\title{
Envelhecimento saudável: uma revisão das pesquisas em Língua Inglesa**
}

Paula J. Gardner

Resumo: "Envelhecimento saudável", como uma perspectiva particular para explorar o relacionamento entre saúde e envelhecimento, é um paradigma teórico poderoso na cultura e prática da gerontologia e uma força dominante nas agendas de pesquisa, organizações financiadoras e governos no mundo de língua inglesa. Escrito a partir de uma perspectiva norte-americana, essa resenha proporciona um resumo da extensa documentação em literatura de língua inglesa dedicada ao "envelhecimento saudável", incluindo a identificação de críticas-chave e desafios da área. A seção final do trabalho considera o quanto um foco no "ir e vir" ("going and doing") de idosos entre determinados lugares de envelhecimento representa uma direção nova e estimulante para as pesquisas futuras sobre envelhecimento saudável.

Palavras-chave: Saúde do idoso. Geriatria. Envelhecimento da população.

\section{Introdução}

O envelhecimento populacional e as conseqüências desta mudança demográfica estão recebendo atenção crescente em níveis local, nacional e internacional. Embora a busca pela compreensão do significado de velhice remonte às obras de filósofos como Platão e Cícero (CÍCERO, 106 a.C.), em grande parte do mundo de língua inglesa o interesse pelo envelhecimento começou a florescer nos anos 90 em função de vários fatores inter-relacionados. Esses fatores incluem o ambiente político-econômico de países ocidentais de alta renda per capita, a identificação do acentuado e crescente envelhecimento populacional nesses países e o efeito (real e potencial) dessa mudança demográfica nas estruturas soci-

M.A. (PhD Candidate) Department of Public Health Sciences and Collaborative Program Aging and the Life Course. University of Toronto.

Movimento, Porto Alegre, v.12, n. 02, p. 69-92, maio/agosto de 2006. 
ais, incluindo famílias, comunidades, economia, planejamento, desenvolvimento, religião, ambiente, política, cultura e cuidados de saúde. Na América do Norte, bem como muitos outros países de língua inglesa, incluindo a Grã-Bretanha, a atenção tem se voltado para o "envelhecimento saudável" - um movimento contemporâneo que está predominantemente preocupado com a correlação saúde-envelhecimento e, de modo mais específico, com os determinantes de uma "boa velhice".

Uma revisão da literatura internacional anglo-saxã revela que "envelhecimento saudável" é um poderoso paradigma teórico na cultura e prática da gerontologia atual e uma força dominante nas agendas de pesquisa, nas organizações financiadoras e governos (MINKLER, 2002; SCHEIDT, HUMPHERYS; YORGASON, 1999; MOODY, 2001). Enquanto movimento teórico, o envelhecimento saudável sustenta-se no pressuposto de que os processos de envelhecimento são, até certo ponto, modificáveis, o que leva pesquisadores da área a adotarem uma abordagem multidimensional e micro (individual) para compreenderem a relação entre envelhecimento e saúde em seus aspectos biológicos, físicos e sociais. $\mathrm{O}$ envelhecimento saudável é definido através de uma série de critérios (tais como satisfação de vida e disposição de espírito) e medidas objetivas (tais como morbidez e mortalidade) e a definição mais proeminente o descreve como sendo a habilidade de manter três características chave: baixo risco de doença e deficiências relacionadas à doença, alta atividade mental e física e envolvimento ativo na vida cotidiana (ROWE; KAHN, 1998, p. 38).

A crescente atenção à população envelhecente é um fenômeno internacional. Estamos no limiar de uma grande transição demográfica que está estimulando discussão e debates a respeito da velhice nos mais destacados meios culturais, econômicos, políticos e acadêmicos (MOODY, 2001, p.175). Esse limiar no qual nos encontramos proporciona uma extraordinária oportunidade para debates ideológicos, aprofundamento da compreensão, desenvolvimento de teorias, defesa de mudanças e promoção de práticas

Movimento, Porto Alegre, v.12, n. 02, p. 69-92, maio/agosto de 2006 
enquanto forjamos novos caminhos para a compreensão da relação entre saúde e envelhecimento. Neste contexto, essa resenha trata, resumidamente, da produção em língua inglesa dedicada ao "envelhecimento saudável", e inclui a identificação de críticas chave e desafios enfrentados pelo campo. A seção final da resenha considera o quanto um foco no movimento de idosos entre lugares de envelhecimento representa uma direção nova e estimulante para as futuras pesquisas de envelhecimento saudável.

20 que significa "saudável" e "envelhecimento"? Termos e terminologia

Como uma alternativa ao paradigma "perda e declínio", muito popular durante grande parte da curta história da gerontologia, o envelhecimento bem-sucedido é descrito como uma "nova" abordagem (ESTES; BIGGS; PHILLIPSON, 2003) ou "novo" paradigma teórico (HOLSTEIN; MINKLER, 2003; MINKLER; FADEM, 2002; MOODY, 2001) na geriatria e gerontologia. Nesta perspectiva, vários termos foram adotados e frequientemente vêm sendo empregados de forma alternada na literatura do campo: "envelhecimento saudável" (BRYANT, CORBETT; KUTNER, 2001, CUSACK; THOMPSON; ROGERS, 2003; MICHAEL; COLDITZ; COAKLEY; KAWACHI, 1999), "envelhecimento bem-sucedido" (MOEN; DEMPSTER-MCCLAIN; WILLIAMS, 1992; ROWE; KAHN, 1998; RYFF, 1989; STRAWBRIDGE; COHEN; SHEMA; KAPLAN, 1996), "envelhecimento produtivo" (BUTLER; GLEASON, 1985, RANZIJN, 2002) e "bem-estar" (CLARKE; MARSHALL; RYFF; ROSENTHAL, 2000, RUFFING-RAHAL; BARIN; COMBS, 1998). ${ }^{1}$

\footnotetext{
O conceito de envelhecimento produtivo tem uma origem distinta do conceito de enveIhecimento bem-sucedido, apesar de sua semelhança conceitual. Envelhecimento produtivo é visto como a manutenção de um foco específico na utilidade econômica, atividade produtiva e contribuição social e econômica de adultos idosos (ver Butler; Gleason, 1985 Butler; Schecheter, 1995). O envelhecimento produtivo também é regularmente confundido com o "envelhecimento bem-sucedido", dada a semelhança entre produtividade e sucesso na cultura ocidental. Neste trabalho eles são apresentados como sinônimos.
}

Movimento, Porto Alegre, v.12, n. 02, p. 69-92, maio/agosto de 2006. 
O foco deste trabalho são os dois conceitos intimamente ligados "envelhecimento saudável" e "envelhecimento bem-sucedido". Buscas nas bases de dados PsychInfo, Medline, AgeLine, e Web of Science usando as palavras-chave "envelhecimento bem-sucedido" e "envelhecimento saudável" em inglês revelam centenas de artigos publicados para cada um desses dois termos, e produziram a maioria de fontes para esta revisão.

\section{Teorias e modelos chave}

Desde o começo dos anos 60, várias teorias sobre envelhecimento saudável foram propostas incluindo a da atividade, do ócio, da continuidade e sócio-ambiental (CHAPMAN, 2005; NUSSBAUM; PECCHIONI; ROBINSON; THOMPSON, 2000). O Modelo de Envelhecimento Bem-sucedido (ROWE; KAHN, 1997, 1998) e o Modelo de Otimização Seletiva e Compensação (em inglês SOC) (BALTES; BALTES, 1990) são, entretanto, os modelos mais populares e influentes de pesquisa e prática de envelhecimento saudável.

Baseado no reconhecimento da heterogeneidade no processo de envelhecimento, o Modelo de Envelhecimento Bem-sucedido de Rowe e Kahn $(1997,1998)$ é uma estrutura multidimensional composta de três componentes ordenados hierarquicamente que definem envelhecimento saudável como " [...] a habilidade de manter três comportamentos ou características chave: baixo risco de doença e deficiência relacionada à doença, alta atividade mental $\mathrm{e}$ física e envolvimento na vida cotidiana" (KAHN, 1998, p. 38). O Modelo de Envelhecimento Bem-sucedido centra-se no pressuposto de que características "usuais" de envelhecimento são modificáveis ou, dito de outro modo, de que fatores externos (tais como estilo de vida) desempenham um papel significante na determinação de risco na velhice.

O Modelo de Otimização Seletiva e Compensação (SOC) é um modelo psicológico que consiste em três estratégias adaptativas

Movimento, Porto Alegre, v.12, n. 02, p. 69-92, maio/agosto de 2006. 
interrelacionadas: seleção, otimização e compensação (BALTES; BALTES, 1990, BALTES; CARTENSEN, 1996; FREUND; BALTES, 1998). O elemento de "seleção" se refere à perda ou restrição de certas funções associadas ao envelhecimento e à tarefa adaptativa de se concentrar nesses domínios, que são altamente prioritários de acordo com exigências ambientais, motivações individuais e capacidade biológica. A "Otimização" está relacionada com o aumento e enriquecimento de reservas pessoais e maximização da duração da vida quantitativa e qualitativamente. Por fim, "compensação" é o processo pelo qual os indivíduos usam elementos mentais e tecnológicos para compensar perdas funcionais derivadas do envelhecimento. Apesar de não ser tão popular quanto o modelo de Rowe e Kahn, esse modelo traz uma importante contribuição ao discurso de envelhecimento saudável - em vez de ver o envelhecimento bem-sucedido como um estado, o Modelo SOC trata o envelhecimento bem-sucedido como um processo de adaptação contínua. Essa perspectiva enfatiza o desenvolvimento humano e apóia a pesquisa sobre a trajetória de vida.

\section{Achados chave}

A maior parte da literatura em inglês sobre envelhecimento saudável pode ser organizada em duas categorias - biomédica (focada no estado de saúde e preocupada primeiramente com doenças, deficiências e padrões clínicos) e psicossocial (focada no estado mental e ajustes ao processo de envelhecimento). Muito do que se sabe a respeito de envelhecimento saudável, a partir de uma perspectiva biomédica, é baseado em dados de grandes estudos longitudinais como o Estudo Longitudinal de Envelhecimento de Duke (PALMORE, 1979); Estudo Longitudinal de Envelhecimento de Manitoba (ROOS; HAVENS, 1991); o Estudo de Envelhecimento de Alameda County (GURALNIK; KAPLAN, 1989, STRAWBRIDGE; COHEN; SHELMA; KAPLAN, 1996); e o Estudo de Envelhecimento Bem-sucedido de MacArthur (SEEMAN;

Movimento, Porto Alegre, v.12, n. 02, p. 69-92, maio/agosto de 2006. 
LUSIGNOLO; ALBERT; BERKMAN, 2001). A maior parte dessas pesquisas foi realizada nos Estados Unidos, apesar de haver alguns trabalhos de outros países como Canadá, China e Austrália.

Os resultados de tais estudos demonstram que vários fatores chave podem ser organizados em três grupos: demográficos, físicos e apoio ou redes sociais. As características demográficas significativamente associadas ao envelhecimento bem-sucedido são idade, sexo e status socioeconômico. Declínio relacionado à idade foi observado em quase todos os estudos (CHOU; CHI, 2002, LAMB; MYERS, 1999; GARFEIN; HERZOG, 1995, O'ROURKE; MACLENNAN; HADJISTAVROPOULOS; TUOKKO, 2000). O envelhecimento dos homens foi considerado mais bem-sucedido do que o das mulheres (CHOU; CHI, 2002, FORD et al.., 2000, LAMB; MYERS, 1999, STRAWBRIDGE et al., 1996). O status socioeconômico, mensurado primeiramente de acordo com escolaridade, também foi identificado como um determinante importante (GURALNIK; KAPLAN, 1989, O'ROURKE et al., 2000; VAILLANT; VAILLANT, 1990, VAILLANT; WESTERN, 2001).

Os resultados ainda apontam para dois comportamentos, consumo de tabaco e atividade física, como os fatores físicos mais importantes associados ao envelhecimento saudável. Eles claramente demonstram que indivíduos que não fumaram ou abandonaram o cigarro antes dos 50 anos tinham mais propensão a envelhecer de forma bem-sucedida (BURKE et al., 2001, FORD et al., 2000, GURALNIK; KAPLAN, 1989; MICHAEL et al., 1999; PALMORE, 1979, REED et al., 1998; VAILLANT; VAILLANT, 1990). A atividade física mostrou-se um determinante de envelhecimento saudável, tanto em estudos seccionais quanto longitudinais (MENEC, 2003, MICHAEL et al., 1999; STRAWBRIDGE et al., 1996; VAILLANT;VAILLANT, 1990). Outros fatores físicos que se mostraram importantes foram a auto-avaliação do estado de saúde (CHOU; CHI, 2002, GARFEIN; HERZOG, 1995, ROOS; HAVENS, 1991), não ser obeso (GURALNIK; KAPLAN, 1989,

Movimento, Porto Alegre, v.12, n. 02, p. 69-92, maio/agosto de 2006. 
MICHAEL et al., 1999; REED, et al., 1998) e baixa pressão sangüínea (GURALNIK; KAPLAN, 1989, REED et al., 1998, STRAWBRIDGE et al., 1996). Pesquisas realizadas a partir de uma perspectiva biomédica também demonstram que o suporte social é um importante determinante de envelhecimento saudável. Visitas freqüentes (GARFEIN; HERZOG, 1995) e sistemáticas (VAILLANT; VAILLANT, 1990) à família, ter 5 ou mais contatos pessoais (STRAWBRIDGE, et al., 1996), incluindo contatos por telefone, (GARFEIN; HERZOG, 1995) e participar em atividades de grupo (PALMORE, 1979) foram os determinantes mais salientes. Além disso, o apoio de uma rede de pessoas com as quais se têm relacionamentos sociais foi associado à maior quantidade de atividade física (MICHAEL et al., 1999) e cognitiva (SEEMAN et al., 2001). Seeman e colegas (2001) especulam que as redes sociais possam operar como um fator protetor do envelhecimento cognitivo.

A pesquisa psicossocial dedicada ao envelhecimento saudável amplia-se e diversifica-se à medida que os pesquisadores consideram em seus estudos não apenas quais fatores são associados ao envelhecimento saudável, mas também como esses mesmos fatores operam. O conjunto desse trabalho está menos preocupado com a função ou ausência de doenças (os dois componentes privilegiados pela perspectiva biomédica) e mais preocupado com o envolvimento na vida cotidiana. Uma revisão da literatura revela vários achados importantes, sendo que o mais significativo está na área do apoio social e das redes sociais. Estudos revelam não só que o apoio social é um fator determinante no envelhecimento saudável, mas que a sua ampliação ao longo do tempo e redes sociais de apoio pré-estabelcidas estão relacionadas ao bem-estar (GURUNG, TAYLOR;SEEMAN, 2003). Outros estudos também revelam que apoio social tem especificidades de gênero - os homens recebem mais apoio de suas esposas, enquanto mulheres recebem a maior parte da assistência de amigos e parentes (GURUNG; TAYLOR; SEEMAN, 2003). Em um estudo longitu-

Movimento, Porto Alegre, v.12, n. 02, p. 69-92, maio/agosto de 2006. 
dinal sobre a saúde das mulheres, a integração social (definida como a realização de múltiplos papéis sociais) mostrou-se promotora de envelhecimento saudável (MOEN; DEMPSTERMCCLAIN;WILLIAMS, 1992). A pesquisa de Day e Day (1993) a respeito de mulheres idosas revelou que os níveis de bem-estar eram maiores entre as que viviam apenas com o marido ou sozinhas, e menores entre as que viviam com parentes que não seus maridos. Os autores atribuem essa discrepância à perda de controle pessoal.

Em outra pesquisa a respeito do controle percebido das redes sociais, Bailis e Chipperfield (2002) sugerem que "auto-estima coletiva" (a auto-avaliação do indivíduo como membro de um grupo social) pode proteger a saúde de adultos mais velhos que têm a percepção de pouco controle pessoal sobre a saúde. O efeito positivo de sentir-se parte de uma comunidade é demonstrado em outra pesquisa. Leviatan (1999) descobriu que a vida nos kibbutz, na qual os adultos mais velhos são valorizados como membros importantes e respeitados de uma comunidade, é um sistema social favorável que contribui para o envelhecimento saudável (alta percepção de auto-estima e baixas taxas de mortalidade). Outra pesquisa dentro de uma perspectiva psicossocial concentrou-se na psicologia individual e no efeito da personalidade no envelhecimento saudável. Nesse trabalho se explorou da identidade de gênero, demostrando-se particularmente o relacionamento positivo entre androginia (uma combinação de características que por esteriótipo são consideradas exclusivamente masculinas ou femininas) e envelhecimento saudável (RUFFINGRAHAL;BARIN;COMBS,1998, SHIMONAKA; NAKAZATO; HOMMA, 1996). O trabalho de Shimonaka (1996) a respeito da personalidade sugere que comportamento Tipo B, particularmente a característica de flexibilidade, está ligado à longevidade. Em um estudo a respeito de idosos finlandeses, Uotinen, Sutama e Ruoppila (2003) examinaram o relacionamento entre a identificação da idade (por exemplo, percepções de envelhecimento pessoal) e enve-

Movimento, Porto Alegre, v.12, n. 02, p. 69-92, maio/agosto de 2006. 
lhecimento saudável. Descobertas indicam que aqueles que estão envelhecendo bem mantêm uma identidade mais jovial, uma idade ideal mais alta, maior satisfação com sua idade cronológica, definiram o começo da velhice mais tarde e manifestaram uma disposição de viver até os 100 anos.

\section{Resumo e lacunas}

Independentemente da perspectiva, o principal objetivo da pesquisa de envelhecimento saudável é identificar e explorar os determinantes de uma "boa velhice". O extenso programa de pesquisa dedicado ao envelhecimento saudável revela a multidimensionalidade e complexidade desse fenômeno à medida que situa o envelhecimento muito além da saúde e longevidade. A pesquisa de envelhecimento saudável apóia (e é apoiada por) uma mudança na gerontologia em direção ao reconhecimento da heterogeneidade de idosos e os aspectos positivos da velhice. Cada linha de pesquisa (biomédica e psicossocial) fornece insights únicos e conhecimentos importantes que juntos oferecem uma contribuição significativa para o campo da gerontologia e aos discursos ligados à saúde de idosos. Essa revisão também aponta para várias lacunas dentro da literatura. Apesar do contínuo reconhecimento da multidimensionalidade do envelhecimento saudável, a pesquisa é dominada por perspectivas biológicas e psicológicas. "Envelhecimento" e "saúde" são definidos de forma limitada: há uma concentração na saúde física e habilidade funcional e poucos estudos se centram nos fatores sociais e ambientais associados ao envelhecimento. Há muito poucos esboços de pesquisas qualitativas e muitas populações ausentes na pesquisa, incluindo deficientes, homossexuais, culturas diversas e aqueles menos saudáveis. Uma das lacunas mais evidentes na literatura é a falta de pesquisa sobre aspectos macro-estruturais no envelhecimento, já que a literatura reflete um foco dominante no indivíduo. Ironicamente, apesar desse foco individual, a voz subjetiva e experiência vivida de idosos é quase completamente inexistente nessa literatura.

Movimento, Porto Alegre, v.12, n. 02, p. 69-92, maio/agosto de 2006. 


\section{Desafios e críticas}

O discurso de envelhecimento saudável traz uma contribuição importante e significativa à gerontologia, especificamente ao discutir a relação entre envelhecimento e saúde operacionalizada em teorias e práticas de saúde. Desde o seu princípio, entretanto, o movimento do envelhecimento saudável, e particularmente o Modelo de Envelhecimento Bem-sucedido, têm sido criticados. Primeiramente, alguns autores argumentam que devido ao fato dos participantes terem que atingir todos os três critérios (evitar doenças, manter alta atividade cognitiva e física e envolvimento na vida cotidiana), as pesquisas baseadas no modelo de Rowe e Kahn estão voltadas para um pequeno e elitizado segmento da população (STRAWBRIDGE et al., 1996, p.135). Além disso, o componente "viver sem doença e déficit" tem sido questionado. Vários pesquisadores argumentam que a maioria dos idosos vive com algum grau de dependência ou de doença e, quando perguntados, esperam uma deterioração da sua capacidade física em função da idade cronológica (STRAWBRIDGE; WALLHAGEN;COHEN, 2002, BRYANT; CORBETT; KUTNER, 2001, VON FABER et al., 2001). Nessa perspectiva, a obtenção do status de envelhecimento bem-sucedido parece ser inatingível e não-realista.

As críticas dirigidas especificamente ao modelo de Rowe e Kahn também têm desafiado a pesquisa sobre envelhecimento saudável. Primeiramente, a preocupação com os elementos físicos e biológicos da saúde e envelhecimento tem sido criticada. Essa revisão encontrou suporte para as reivindicações feitas por outros autores (GARFEIN; HERZOG, 1995, p. S77) de que o envelhecimento precoce está predominantemente assentado em termos de saúde física, apesar do seu objetivo multidimensional. Além disso, alega-se que o Modelo de Envelhecimento Bem-sucedido não se dirige às (macro) estruturas sociais que são essenciais para pôr em prática o envelhecimento bem-sucedido (RILEY, 1998). Outras críticas ao envelhecimento saudável se referem à sua

Movimento, Porto Alegre, v.12, n. 02, p. 69-92, maio/agosto de 2006. 
"normatividade implícita" (e, portanto, não percebida) (HOLSTEIN; MINKLER, 2003, p. 791) deste constructo, como por exemplo, os valores (definidos como padrões do desejável, TAYLOR; BENGSTON, 2001, p. 125) sobre os quais os termos "envelhecimento" e "saudável" estão baseados.

Alguns autores argumentam que os valores caucasianos e ocidentais - individualismo, sucesso e produtividade - estão incrustados na ideologia do envelhecimento saudável e assentados em termos de responsabilidade individual, autonomia pessoal e independência (HOLSTEIN; MINKLER, 2003, MOODY, 2001, 2005, TAYLOR; BENGSTON, 2001; TORNSTAM, 1992). Essa abordagem caracteristicamente americana (MOODY, 2005 e TORRES, 1999) põe um enorme peso na "conquista" do envelhecimento bem-sucedido e conduz a uma questão: e aqueles que são incapazes ou até mesmo relutantes a atingir esse status? Em outras palavras, quais as conseqüências de uma perspectiva que é afirmada em valores de sucesso e responsabilidade em um nível individual?

\section{Preenchendo lacunas e respondendo críticas}

Há muitos exemplos de pesquisas que operam fora do que pode ser considerado "pesquisa de envelhecimento saudável tradicional". Pesquisas a partir de "perspectivas alternativas" ajudam a preencher lacunas, responder críticas e fornecem uma nova compreensão da relação entre envelhecimento e saúde. Atualmente, duas áreas de pesquisa oferecem proposições ímpares e apontam para o futuro dos estudos sobre envelhecimento saudável: a) pesquisas sobre a opinião de idosos e b) pesquisas que buscam contextualizar o envelhecimento saudável ao centrarem-se nos "lugares de envelhecimento".

Respondendo as críticas de que a voz dos idosos tem sido negligenciada, o fim dos anos 90 testemunhou o começo da linha de pesquisa relacionada ao envelhecimento saudável baseada na

Movimento, Porto Alegre, v.12, n. 02, p. 69-92, maio/agosto de 2006. 
investigação das opiniões dos próprios idosos. O trabalho investigativo que busca a interpretação dos idosos fornece uma preciosa contribuição sobre o significado subjetivo de conceitos como envelhecimento saudável, a relevância desses conceitos para os idosos e seu grupo etário e como a satisfação com a vida e o envelhecer com saúde são determinados (FISHER, 1992, p. 194). Há duas descobertas significantes e inter-relacionadas nessa área de pesquisa. Primeiramente, os dois atributos-chave do envelhecimento saudável, identificados pelos idosos, são envolvimentos com a vida (também referido como senso de propósito, compromisso ou atividade) e apoio social e relacionamentos (BRYANT; CORBETT; KUTNER, 2001, FISHER; DAY; COLLIER, 1998, FISHER, 1995; FISHER; SPECHT, 1999, KNIGHT; RICCIARDELLI, 2003, PHELAN et al., 2004; TATE; LAH; CUDDY, 2003). O "ir e vir" (going and doing), que freqüentemente envolve o inter-relacionamento entre esses dois atributos-chave, é particularmente relevante em idosos (BRYANT; CORBETT; KUTNER, 2001). Em segundo lugar, os resultados de alguns estudos ilustram que, apesar na hipótese de que muito da pesquisa sobre envelhecimento saudável se baseia na ausência de doença e na manutenção de alta atividade funcional, o envelhecimento saudável pode ocorrer (e ocorre) mesmo na presença de doença, deficiência ou outras privações (BOYLE; COUNTS, 1988, FISHER; SPECHT, 1999, KNIGHT; RICCIARDELLI, 2003; TATE; LAH; CUDDY, 2003).

Resumindo, a pesquisa que busca as opiniões de idosos destaca que a capacidade funcional não pode ser visto como um fim em si mesmo, e sim como um meio para um determinado fim - um fim que está muito mais ligado à possibilidade de participação e envolvimento na vida diária do que à habilidade funcional propriamente dita.

\section{Lugares de envelhencimento}

A pesquisa dedicada à investigação sobre "envelhecimento e lugares" pode dar resposta à descontextualização e particular-

Movimento, Porto Alegre, v.12, n. 02, p. 69-92, maio/agosto de 2006. 
mente à omissão de fatores ambientais associados ao envelhecimento saudável. Descobertas de várias disciplinas, incluindo a gerontologia social, geografia da saúde, psicologia, antropologia social e promoção da saúde, demonstram que o lugar onde se vive é importante tanto para a saúde (status e comportamento) quanto para o envelhecimento (processos e experiências). Como Moon (1995, p. 4) descreve, "Saúde (e similarmente envelhecimento) não existe em um vácuo social, onde processos e experiências não estão situados no tempo ou espaço, desfocados ou deslocados". A importância do lugar na gerontologia é refletida em um crescimento da pesquisa no recém identificado campo interdisciplinar da "gerontologia geográfica" (ANDREWS, et al., 2005). De acordo com Wiles $(2005$, p. 100) a gerontologia geográfica preocupa-se com "[...] as maneiras pelas quais espaço e lugar se relacionam com a experiência e as necessidades dos idosos", e os pesquisadores que atuam dentro dessa perspectiva buscam entender " [...] como o envelhecimento afeta lugares e espaços específicos, e de que modo contextos específicos influenciam em assuntos relacionados a envelhecimento e idosos". No seu ensaio Spaces for inquiry into the role of place for older people's care (sem tradução para o português), Cutchin (2005) descreve "lugar" como um amálgama de aspectos sociais, culturais, históricos, políticos, econômicos e físicos que compõem o contexto significativo da vida humana. Ele argumenta que o lugar é um objeto complexo de pesquisa, vital para o bem-estar dos idosos e oferece muitas avenidas interdisciplinares para o trabalho dos pesquisadores.

Há um discurso diversificado e crescente dedicado aos "lugares de envelhecimento". O corpo, o lar e o asilo são três focos importantes de pesquisas. A geografia do corpo é um foco de interesse emergente. Pesquisas nessa perspectiva abordam primeiramente o "corpo envelhecente" através de sua deterioração e declínio físico-mental (KEARNS; MOON, 2002, MCHUGH, 2003, NAIR, 2005, PARR, 2002). Desenhado dentro de uma perspectiva antropológica com 12 meses de trabalho de campo em um lar para

Movimento, Porto Alegre, v.12, n. 02, p. 69-92, maio/agosto de 2006. 
idosos de baixa renda, Kontos (2000, p. 269) explora a "dinâmica relação entre corpo, significado e lugar". A sua pesquisa expõe a relação entre cultura e biologia, criticando afirmações de experiências e significados universais de velhice. Kearns e Andrews (2005) sugerem que, além de dar continuação à pesquisa a respeito do corpo envelhecente, os discursos sobre envelhecimento e o corpo como um lugar podem fornecer uma importante orientação para o estudo sobre a corporeidade envelhecente em contextos positivos tais como sexualidade, estado físico e espiritualidade.

Entretanto, a maior parte da pesquisa sobre lugares de envelhecimento centra-se em dois ambientes: o lar e os serviços de atenção ao idoso. A pesquisa que toma os lares como foco aponta que esse é um lugar de muita influência na saúde e bem-estar de idosos. Kontos (2000) argumenta que o lar é um lugar importante para a pesquisa sobre o envelhecimento, já que desempenha um papel crítico na manutenção de um senso de identidade pessoal do idoso. O lar, ao contrário de outros espaços de residência, não compromete a independência do idoso. Outra pesquisa que toma o lar como um lugar de envelhecimento procura centrar as análises no sentimento de apego ao local. Cutchin, Owen e Chang (2003) conduziram uma pesquisa empírica para explorar a importância do apego, envolvimento social não-familiar, valorização e características individuais no processo de "sentir-se em casa" em locais de assistência a pessoas dependentes. Os resultados demonstram que o apego a um lugar na comunidade é necessário, mas não suficiente para dar conta do processo de "adaptação" (sentir-se em casa). O envolvimento social não-familiar também desempenha um papel fundamental nesta "adaptação", tanto o apreço pelo lugar quanto o envolvimento social não-familiar exibem efeitos positivos no resultado de "sentir-se em casa". Pesquisas sobre moradias individuais e asilos estão preocupadas, sobretudo, com o que tem sido chamado de debate "cuidado domiciliar versus cuidado comunitário" (ANDREWS; PHILLIPS, 2002).

Movimento, Porto Alegre, v.12, n. 02, p. 69-92, maio/agosto de 2006. 
Mais recentemente, entretanto, as "lentes" da pesquisa centraram o foco na compreensão das nuances das experiências dos idosos, mais especificamente dos significados associados a elas nesses locais, e suas implicações para o bem-estar. Andrews et al (2005) refletem de que modo um foco conceitual sobre o lugar pode informar a prática de enfermagem. A análise e discussão empregadas nesse estudo, bem como nos trabalhos de outros pesquisadores, levam os autores a refletir sobre uma complexa relação entre práticas humanas e ambientes físicos, "lugares" que ganham sentido social, cultural e simbólico e que, por essa razão, tornam-se importantes locais de apego e significação pessoal. A partir de uma perspectiva prática eles sugerem que um foco de investigação centrado em conceitos gerontológicos fundamentais (como autonomia, qualidade de vida e cuidado) para entender os diferentes modos de "lugar", pode dar uma importante contribuição na melhora das instituições que oferecem cuidados de longa duração.

Os resultados dessas "abordagens alternativas" na pesquisa sobre o envelhecimento saudável revelam dois fatores importantes e inter-relacionados. Primeiramente, a pesquisa que busca a opinião dos idosos ilustra que o "ir e vir" (going and doing) é um importante determinante do envelhecimento saudável. Em segundo lugar, o papel significativo do lugar no processo e nas experiências relativas ao envelhecimento ganha destaque. A investigação desenvolvida a partir desses conceitos fornece novos insights e passa a ser tratada como uma importante contribuição à pesquisa e prática de envelhecimento saudável de um modo geral. Sugerese aqui que a pesquisa de envelhecimento saudável preocupada fundamentalmente com o "ir e vir" ("going and doing") de idosos em seus locais de convívio abre perspectivas promissoras para futuros estudos na área.

Movimento, Porto Alegre, v.12, n. 02, p. 69-92, maio/agosto de 2006. 


\section{8 À espera: Envelhecimento saudável e o "movimento" entre "lugares de envelhecimento"}

A pesquisa que busca a compreensão das transições e migrações entre lugares de envelhecimento, bem como o impacto desse movimento na saúde dos idosos, representa um novo e interessante campo de estudos para a pesquisa sobre envelhecimento saudável. Apesar de limitadas, duas abordagens que exploram esse fenômeno foram identificadas. A pesquisa de gerontologia crítica sobre comunidades de aposentados como "espaços sociais de envelhecimento" e representa uma tentativa de refletir sobre o movimento de idosos entre lugares de envelhecimento. Katz (2005) e outros (LAWS, 1995, MCHUGH, 2003) examinaram comunidades de aposentados, e em particular as comunidades de "Sun City" na Flórida, considerando-as um interessante "espaço de envelhecimento" no qual eles exploram as experiências de vida de pessoas numa cultura particular de envelhecimento transitório. A partir de uma perspectiva pós-moderna, as suas análises ilustram uma "espacialidade de envelhecimento" onde as relações entre idosos e os espaços e locais em que vivem revelam atitudes societais e valores profundamente enraizadas (MCHUGH, 2003). Autores que trabalham a partir de uma perspectiva de gerontologia crítica destacam a relação entre lugares migratórios (comunidades de aposentados), "imagens anti-envelhecimento" e políticas de envelhecimento dirigidas ao consumidor que servem para fragmentar identidades no transcorrer da vida e nesses cenários.

Alternativamente, um recente estudo britânico Environment and Identity in Later Life: a cross-setting study (PEACE; HOLLAND; KELLAHER, 2005, sem título em português) revela uma nova compreensão do significado de movimento entre lugares de envelhecimento. No seu amplo estudo, os pesquisadores prestaram considerável atenção às "jornadas" que os participantes da pesquisa faziam quando se deslocavam de seus locais preferidos nas suas casas. Essas "jornadas" podem ser simplesmente de

Movimento, Porto Alegre, v.12, n. 02, p. 69-92, maio/agosto de 2006. 
um aposento para outro na casa ou podem envolver deslocar-se à vizinhança próxima (dentro de uma distância de caminhada). Vinte homens e trinta e quatro mulheres entre 61 e 93 anos de idade que viviam em vários tipos de ambientes (incluindo apartamentos em condomínios com assistência, lares de cuidado residencial, casas e fazendas) foram entrevistados em três locais (de rurais a urbanos). Os resultados ilustram que, para os idosos que vivem em sua própria casa (não lares assistenciais), as vizinhanças próximas (por exemplo, dentro de uma distância de caminhada) eram muito importantes para a saúde e bem-estar (físico, psicológico e social) dos participantes. pendente é um estágio crítico na construção da identidade porque, sem os contextos mais amplos que existem além da residência, o lar em si se diminui como uma fonte de construção de identidade. A capacidade contínua de se envolver com "o outro" é representada pela vizinhança de uma forma que uma residência isoladamente não pode demonstrar ou provar [...] Resultados de estudos confirmam uma espacialidade de envelhecimento descrita por Laws (1995; 1997), e ilustram que o entrosamento real em vizinhanças materiais $e$ sociais é essencial para o bem-estar e identidade própria (PEACE; HOLLAND; KELLAHER, 2005, p. 202- 203).

Para finalizar, cabe retomar alguns dos pontos anteriormente mencionados. A literatura anglo-saxã internacional sobre o "envelhecimento saudável" faz uma significativa contribuição para a saúde e bem-estar dos idosos ao revelar tendências teóricas e descrever práticas atuais que se centram nos aspectos positivos da velhice, propondo que se considere a promoção à saúde e prevenção de doenças para esse grupo etário. Muito da pesquisa nessa área, entretanto, é limitada em seu escopo teórico e metodológico, pois negligencia importantes fatores sociais e ambientais associa-

Movimento, Porto Alegre, v.12, n. 02, p. 69-92, maio/agosto de 2006. 
dos com a saúde e bem-estar na velhice. Particularmente, muitos dos trabalhos sobre envelhecimento saudável falham por não contextualizar efetivamente os processos e experiências de envelhecimento e pouco fazem para explorar a experiência subjetiva dos idosos. Não obstante, pesquisas inovadoras se propõem a ir além dos limites do "envelhecimento saudável tradicional" para desenvolver maneiras alternativas de explorar a relação entre saúde e envelhecimento. Alguns dos resultados desses estudos alternativos sugerem que a pesquisa de envelhecimento saudável que se centra no "ir e vir" ("going and doing") de idosos nos entre lugares de envelhecimento representa uma perspectiva nova e interessante para futuras pesquisas.

Aging Health: a review in the research's
English language
Abstract: "Aging Health", as a particular
perspective to explore the relationship between
health and aging, is a powerful theoretical paradigm
in the practical culture and of the gerontology and a
dominant force in research agendas, organizations
financiers and governments in the world of English
language. Writing from a North American
perspective, this summary provides a summary of
the extensive documentation in literature of
dedicated English language to the "aging health",
including the identification of critical-key and
challenges of the area. The final section of the work
considers how much a focus in "going and coming"
("going and doing") of aged between determined
aging places represents a new direction and
stimulant for the future research overageing health.
Keywords: Aging health. Geriatrics. Demographic
aging.


Envejecimeiento saludable: una revisión de las pesquisas en lengua inglesa.

Resumen: "Envejecimiento saludable", con una perspectiva particular para explorar la relación entre salud y envejeciemento, es un paradigma teórico poderoso en la cultura e práctica de la geriatria y una fuerza dominante en las agendas de pesquisa, organizaciones financieras y gobiernos en el mundo de la lengua inglesa. Escrito a partir de una perspectiva norte-americana, esa reseña proporciona un resumen de la extensa documentación en literatura en la lengua inglesa dedicada ao "envejecimiento saludable", incluso la identificación de las críticas-clave e desafíos de la área. La sección final del trabajo considera o cuanto un foco en el "ir y venir" ("going and doing") del ancianos entre determinados lugares de envejecimiento representa una dirección nuova e incitante para las pesquisas futuras sobre envejecimiento saludable. Palabras-clave: Salud del anciano. Geriatria. Envejecimiento de la población.

\section{REFERÊNCIAS}

ANDREWS, G. J.; HOLMES, D.; POLAND, B.; LEHOUX, P.;MILLER, K. L.; PRINGLE, D. Airplanes are flying nursing homes: geographies in the concepts and locales of gerontological nursing practice. Journal of Clinical Nursing, v. 14, p.109120, 2005.

ANDREWS, G. J.; PHILLIPS, D. R. Changing local geographies of private residential care for older people 1983-1999: lessons for social policy in England and Wales. Social Science \& Medicine, v. 55, n.1, p. 63-78, 2002.

ANDREWS, G.; KEARNS, R.; KONTOS, P., WILSON, V. (forthcoming). Their finest hour: older people, oral histories, and the historical geography of social life. Socia \& Cultural Geography, v.7, n. 2, p.153-177, 2006.

BAILIS, D. S.; CHIPPERFIELD, J. G. Compensating for losses in perceived personal control over health: A role for collective self-esteem in healthy aging. Journal of Gerontology Series B-Psychological Sciences and Social Sciences, v. 57, n.6, p. P531-539, 2002.

BALTES, M. M.; CARTENSEN, L. L. The process of successful aging. Aging and Society, v. 15, p. 397-422, 1996.

Movimento, Porto Alegre, v.12, n. 02, p. 69-92, maio/agosto de 2006. 
BALTES, P. B.; BALTES, M. M. Successful aging: Perspectives from the behavioral sciences. New York: Cambridge University, 1990.

BOYLE, J. S.; COUNTS, M. M. Toward Healthy Aging - a Theory for CommunityHealth Nursing. Public Health Nursing, v. 5, n. 1, p. 45-51,1988.

BRYANT, L. L., CORBETT, K. K.,; KUTNER, J. S. In their own words: a model of healthy aging. Social Science \& Medicine, v. 53, n.7, p.927-941, 2001.

BURKE, G. L., ARNOLD, A. M., BILD, D. E., CUSHMAN, M., FRIED, L. P., NEWMAN, A., et al.. Factors associated with healthy aging: The cardiovascular health study. Journal of the American Geriatrics Society, v. 49, n.3, p. 254-262, 2001.

BUTLER, R. N.; GLEASON, H. P.. Productive aging. New York: Springer, 1985.

BUTLER, R. N.; SCHECHTER, M. Productive aging. In: MADDOX, G. (Ed.). The encyclopedia of aging. 2nd ed.. New York: Springer, p. 763-764,1995.

CHAPMAN, S. A. Theorizing about aging well: Constructing a narrative. Canadian Journal of Public Health-Revue Canadienne de Sante Publique, v. 24, n.1, p.9-18, 2005.

CHOU, K. L.; CHI, I. Successful aging among the young-old, old-old, and oldest-old Chinese. International Journal of Aging \& Human Development, v. 54, n.1, p. 1-14, 2002.

CICERO. On old age. Tranlated E. Shuckburgh. New York: Colliers \& Son, 19091914. v. 9, p. 2. (The Harvard Classics)

CLARKE, P. J.; MARSHALL, V.; RYFF, C. D.; ROSENTHAL, C. J. Well-being in Canadian seniors: Findings from the Canadian study of health and aging. Canadian Journal on Aging-Revue Canadienne Du Vieillissement, v.19, n.2, p. 139-159, 2000.

CUSACK, S. A.; THOMPSON, W. J. A.;ROGERS, M. E. Mental Fitness for Life: Assessing the impact of an 8-week mental fitness program on healthy aging. Educational Gerontology, v.29, n.5, p.393-403, 2003.

CUTCHIN, M. P. Spaces for inquiry into the role of place for older people's care. Journal of Clinical Nursing, v.14, p.121-129, 2005.

CUTCHIN, M. P.; OWEN, S. V.;CHANG, P. J. Becoming "at Home" in assisted living residences: Exploring place integration processes. Journal of Gerontology, $v$. 58B, n.4, p.S234-S243, 2003.

DAY, A. T.; DAY, L. H. Living Arrangements and Successful Aging among EverMarried American White Women 77-87 Years of Age. Aging and Society, 13, 365387, 1993.

DYCK, I.; KONTOS, P.; ANGUS, J.;MCKEEVER, P. The home as a site for longterm care: meanings and management of bodies and spaces. Health \& Place, v.11, n.2, p.173-185, 2005. 
ESTES, C. L.; BIGGS, S.;PHILLIPSON, C. Social theory, social policy and aging: A critical introduction. Berkshire: Open University, 2003.

FISHER, B. J. Successful Aging and Life Satisfaction - a Pilot-Study for Conceptual Clarification. Journal of Aging Studies, v.6, n.2, p.191-202, 1992.

FISHER, B. J. Successful aging, life satisfaction, and generativity in later life. International Journal of Aging \& Human Development, v.41, n.3, p.239-250 1995.

FISHER, B. J.; DAY, M.; COLLIER, C. E. Successful aging: Volunteerism and generativity in later life. In: REDBURN, D. E.; MCNAMARA, R. P. (Ed.). Social Gerontology. Westport, CT: Auburn House, p. 43-54, 1998.

FISHER, B. J.; SPECHT, D. K. Successful aging and creativity in later life. Journal of Aging Studies, v.13, n. 4, p.457-472, 1999.

FORD, A. B.; HAUG, M. R.; STANGE, K. C.; GAINES, A. D.; NOELKER, L. S.; JONES, P. K.. Sustained personal autonomy: A measure of successful aging. Journal of Aging and Health, v.12, n.4, p. 470-489, 2000.

FREUND, A. M.; BALTES, P. B. Selection, optimization, and compensation as strategies of life management: Correlations with subjective indicators of successfu aging. Psychology and Aging, v.13, n.4; p.531-543, 1998.

GARFEIN, A. J.; HERZOG, A.; ROBUST, R; Aging among the Young-Old, Old-Old, and Oldest-Old. Journals of Gerontology Series B-Psychological Sciences and Social Sciences, v. 50, n.2, p. S77-S87, 1995.

GURALNIK, J. M.; KAPLAN, G. A. Predictors of Healthy Aging - Prospective Evidence from the Alameda County Study. American Journal of Public Health v.79, n.6, p. 703-708,1989

HOLSTEIN, M. B.; MINKLER, M. Self, society, and the "new gerontology". The Gerontologist, v.43, n.6, p.787-796, 2003.

KATZ, S. Cultural aging: Life course, lifestyle, and senior worlds. Peterborough: Broadview, 2005.

KEARNS, R. A.; ANDREWS, G. J. Placing aging: positionings in the study of older people. In: Andrews, G. J.; Phillips, D. (Ed.). Aging and place: Perspectives, policy, practice. New York: Routledge, p. 13-23, 2005.

KEARNS, R.; MOON, G. From medical to health geography: novelty, place and theory after a decade of change. Progress in Human Geography, v. 26, n. 5, p.605-625, 2002.

KNIGHT, T; RICCIARDELLI, L. A. Successful aging: Perceptions of adults aged between 70 and 101 years. International Journal of Aging \& Human Development, v. 56, n. 3, p 223-245, 2003. 
KONTOS, P. Resisting institutionalization: Constructing old age and negotiating home. In: GUBRIUM, J.; HOLSTEIN, J. A. (Ed.). Aging and everyday life. Oxford: Blackwell, p. 255-272, 2000.

LAMB, V. L.; MYERS, G. C. A comparative study of successful aging in three Asian countries. Population Research and Policy Review, v.18, n. 5, p. 433-449, 1999.

LAWS, G. Embodiment and emplacement: Identities, representation and landscape in sun city retirement communities. International Journal of Aging \& Human Development, v. 40, n.4, p. 253-280, 1995.

LAWS, G. Spatiality and age relations. In: JAMIESON, A.; HARPER, S.; VICTOR, C. (Ed.). Critical approaches to ageing and later life. Buckingham: British Society of Gerontology Conference, p. 90-101, 1997.

LEVIATAN, U. Contribution of social arrangements to the attainment of successful aging - The experience of the Israeli Kibbutz. Journals of Gerontology Series BPsychological Sciences and Social Sciences, v.54, n. 4, p.P205-P213, 1999.

MCHUGH, K. E. Three faces of ageism: society, image and place. Aging \& Society, v.23, p. 165-185, 2003.

MENEC, V. H. The relation between everyday activities and successful aging: A 6year longitudinal study. Journals of Gerontology Series B-Psychological Sciences and Social Sciences, v. 58, n. 2, p.S74-S82, 2003.

MICHAEL, Y. L.; Colditz, G. A.; Coakley, E.; Kawachi, I. Health behaviors, social networks, and healthy aging: Cross-sectional evidence from the Nurses' Health Study. Quality of Life Research, v. 8, n.8, p. 711-722, 1999.

MINKLER, M.; FADEM, P. "Successful Aging:" A disability perspective. Journal of Disability Policy Studies, v.12, n.4, p.229-235, 2002.

MOEN, P.; DEMPSTER-MCCLAIN, D.; WILLIAMS, R. M. Successful Aging - a LifeCourse Perspective on Womens Multiple Roles and Health. American Journal of Sociology, v.97, n.6, p.1612-1638, 1992.

MOODY, H. R. Productive aging and the ideology of old age. In: MORROW, N.; HOWELL, J. HINTERLONG; SHERRADEN, M. (Ed.). Productive Aging. Baltimore: John Hopkins University, 2001. p. 175-196.

MOON, G. (Re)placing research on health and health care. Health \& Place, v.1, p.1-4, 1995.

NAIR, K. The physically aging body and the use of space. In: ANDREWS G.; PHILLIPS, D. (Ed.). Aging and place: perspectives, policy, practice. New York: Routledge, 2005. p. 110-117.

NUSSBAUM, J. F.; PECCHIONI, L. L.; ROBINSON, J. D.; THOMPSON, T. L. Communication and aging. 2nd ed.. Mahwah, NJ: Lawrence Erlbaum, 2000.

O'ROURKE, N.; MACLENNAN, R.; HADJISTAVROPOULOS, T.; TUOKKO, H. Longitudinal examination of the functional status of older adults: Successful aging 
within a representative Canadian sample. Canadian Journal on Aging-Revue Canadienne Du Vieillissement, v.19, n. 4, p. 441-455, 2000.

PALMORE, E. Predictors of Successful Aging. Gerontologist, v.19, n.5, p.427431, 1979.

PARR, H. Medical geography: diagnosing the body in medical and health geography, 1999-2000. Progress in Human Geography, v.26, n. 2, p.240-251, 2002.

PEACE, S. M.; HOLLAND, C.; KELLAHER, L. Making space for identity. In: ANDREWS, G. J.; PHILLIPS, D. (Ed.). Aging and place: perspectives, policy, practice. New York: Routledge, 2005. p. 188-204.

PHELAN, E. A.; LARSON, E. B. "Successful aging" - Where next? Journal of the American Geriatrics Society, v.50, n.7, p.1306-1308, 2002.

RANZIJN, R. Potential of older adults to enhance community quality of life: links between positive psychology and productive aging. Aging International, v. 27, n.2, p. 30-55, 2002.

REED, D. M.; FOLEY, D. J.; WHITE, L. R.; HEIMOVITZ, H.; BURCHFIEL, C. M. MASAKI, K. Predictors of healthy aging in men with high life expectancies. American Journal of Public Health, v. 88, n.10, p.1463-1468,1998.

RILEY, M. Letters to the editor. The Gerontologist, v.38, n.2, p.151, 1998.

ROOS, N. P.; HAVENS, B. Predictors of Successful Aging - a 12-Year Study of Manitoba Elderly. American Journal of Public Health, v.81, n. 1, p.63-68, 1991.

ROWE, J. W.; KAHN, R. L. Human Aging - Usual and Successful. Science, v. 237, n.4811, p.143-149, 1987

ROWE, J. W.; KAHN, R. L. Successful aging. Gerontologist, v. 37, n. 4, p. 433 440, 1997.

ROWE, J. W.; KAHN, R. L. Successful aging. New York: Pantheon, 1998.

RUFFING-RAHAL, M. A.; BARIN, L. J.; COMBS, C. J. Gender role orientation as a correlate of perceived health, health behavior, and qualitative well-being in older women. Journal of Women \& Aging, v.10, n.1, p.3-19, 1998.

RYFF, C. D. Beyond Ponce De Leon and Life Satisfaction - New Directions in Quest of Successful Aging. International Journal of Behavioral Development, v. 12 n. 1, p.35-55, 1989.

SCHEIDT, R. J.; HUMPHERYS, D. R.; YORGASON, J. B. Successful aging: What's not to like? Journal of Applied Gerontology, v.18, n. 3, p.277-282, 1999.

SEEMAN, T. E.; LUSIGNOLO, T. M.; ALBERT, M.; BERKMAN, L. Social relationships, social support, and patterns of cognitive aging in healthy, high-functioning older adults: MacArthur studies of successful aging. Health Psychology, v. 20, n.4, p.243255, 2001.

Movimento, Porto Alegre, v.12, n. 02, p. 69-92, maio/agosto de 2006. 
SHIMONAKA, Y.; NAKAZATO, K.; HOMMA, A. Personality, longevity, and successful aging among Tokyo metropolitan centenarians. International Journal of Aging \& Human Development, v. 42, n.3, p.173-187, 1996.

STRAWBRIDGE, W. J.; COHEN, R. D.; SHEMA, S. J.; KAPLAN, G. A. Successful aging: Predictors and associated activities. American Journal of Epidemiology, v.144, n. 2, p.135-141, 1996.

STRAWBRIDGE, W. J.; WALLHAGEN, M. I.; COHEN, R. D. Successful aging and well-being: Self-rated compared with Rowe and Kahn. Gerontologist, v. 42, n. 6 , p. 727-733, 2002.

TATE, R. B.; LAH, L.; CUDDY, T. E. Definition of successful aging by elderly Canadian males: The Manitoba follow-up study. Gerontologist, v.43, n. 5, p. 735-744, 2003.

TAYLOR, B.; BENGSTON, V. Sociological perspectives on productive aging. In: MORROW-HOWELL, N.; HINTERLONG, J.; SHERRADEN, M. (Ed.). Productive Aging. Baltimore: John Hopkins University, 2001. p. 121-144.

TORNSTAM, L. The Quo-Vadis of Gerontology - on the Scientific Paradigm of Gerontology, v.32, n. 3, p. 318-326, 1992.

TORRES, S. A. culturally-relevant theoretical framework for the study of successful aging. Ageing \& Society, v.19, p. 33-51, 1999.

UOTINEN, V.; SUUTAMA, T.; RUOPPILA, I. Age identification in the framework of successful aging. A study of older Finnish people. International Journal of Aging \& Human Development, v.56, n. 3, p.173-195, 2003.

VAILLANT, G. E.; VAILLANT, C. O. Natural-History of Male Psychological Health: A 45-Year Study of Predictors of Successful Aging at Age 65. American Journal of Psychiatry, v. 147, n.1, p.31-37, 1990.

VAILLANT, G. E.; WESTERN, R. J. Healthy aging among inner-city men. International Psychogeriatrics, v.13, n. 4, p.425-437, 2001.

VON FABER, M.; BOOTSMA-VAN DER WIEL, A.; VAN EXEL, E.; GUSSEKLOO, J.; LAGAAY, A. M.; VAN DONGEN, E. et al. Successful aging in the oldest old - Who can be characterized as successfully aged? Archives of Internal Medicine, v.161, n. 22, p.2694-2700, 2001.

WILES, J. Conceptualizing place in the care of older people: the contributions of geographical gerontology. Journal of Clinical Nursing, v.14, p.100-108, 2005.

Recebido em: 10/04/2006

Aprovado em: 20/07/2006

"Tradução de Eduardo Stigger. Revisão técnica de Alex Branco Fraga (ESEF/UFRGS) e Denise Gastaldo (University of Toronto).

Movimento, Porto Alegre, v.12, n. 02, p. 69-92, maio/agosto de 2006. 